\title{
Low-Complexity Iterative Detection and Decoding of Multi-Antenna Systems Employing Channel and Space-Time Codes
}

\author{
Haris Vikalo ${ }^{\dagger}$ and Babak Hassibi ${ }^{\ddagger}$ \\ †Information Systems Lab, Stanford University, CA 94309 \\ $\ddagger$ Department of Electrical Engineering, California Institute of Technology, CA 91125
}

\begin{abstract}
We study multiple antenna systems that employ spacetime modulation schemes and transmit data encoded by powerful channel codes. Decoders for such codes require probabilistic (soft) information about transmitted bits. We use a modification of the Fincke-Pohst algorithm to solve maximum a posteriori detection problem and efficiently approximate soft information. Simulation results that illustrate performance of the proposed system are presented.
\end{abstract}

\section{Introduction}

In [1], [2], it was shown that channel capacity in multiple antenna systems grows linearly with the minimum of the number of transmit and receive antennas. Early multiple antenna communication systems relied on diversity, which is achieved by sending redundant information through a scattering environment and hoping that the message reaches receiver along at least one of the multiple paths ([3]-[5]). However, achieving the promised capacity results requires more sophisticated means of exploiting the spatial diversity. To this end, space-time coding techniques have been developed. Space-time coding is a modulation technique that imposes spatial and temporal correlation onto a transmitted sequence of modulated symbols. In [6], [7], a practical spacetime coding scheme, called V-BLAST (Vertical Bell-Labs Layered Space-Time), has been demonstrated on a system which employs eight transmit and twelve receive antennas. In this scheme, the original data stream is split into substreams which are then transmitted on individual antennas. The receiver employs nulling and cancelling algorithm to decode the originally transmitted data.

In the following years, there has been a tremendous amount of the research activities in the field of space-time code design. For the communication systems where the channel is assumed to be known to the receiver, space-time trellis [8] and space-time block codes [9], [10] have been proposed. There is also a number of available schemes where the channel is not known to the receiver, ([11]-[16]).

Space-time codes, in general, experience complexity difficulties at very high rates and with a large number of transmit antennas. In particular, the computational complexity of the optimal maximum-likelihood receiver for decoding the trellis space-time codes of [8] grows exponentially with either the rate or the number of transmit antennas. Block space-time codes face similar complexity issues in high rate systems. On the other hand, heuristic techniques for receiver design, such as those employed in V-BLAST scheme, provide implementable complexity. However, aside from being suboptimal, V-BLAST decoding scheme presented in [6] does not work with fewer receive than transmit antennas.

There have also been a number of recently proposed multiple antenna schemes that employ powerful channel codes to encode the transmitted data (see, e.g., [17]-[19] and the references therein). In such schemes, the channel decoder require soft information about the bits in the transmitted sequence. The complexity of providing soft information in multi-antenna systems, however, may become rather high.

In this paper, we consider powerful (turbo, LDPC) channel codes for data encoding in multiple antenna systems that employ space-time codes. In particular, we focus on recently proposed linear dispersive (LD) space-time codes [20]. We show that the LD codes allow for an efficient MAP detection and approximation of the soft information via modified Fincke-Pohst algorithm. The simulation results illustrate excellent performance of the proposed scheme.

\section{System model and linear dispersion codes}

The system model is shown in Figure 1. The information data is first protected embedding redundancy via errorcorrecting codes and then space-time coded and sent across the channel. Receiver uses FP-MAP algorithm to estimate soft information of the transmitted coded sequence and performs joint iterative detection and decoding.

We shall assume a narrow-band, flat-fading multiple an- 
Transmitter:

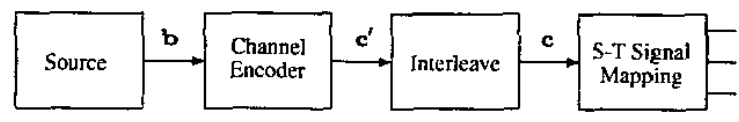

Receiver:

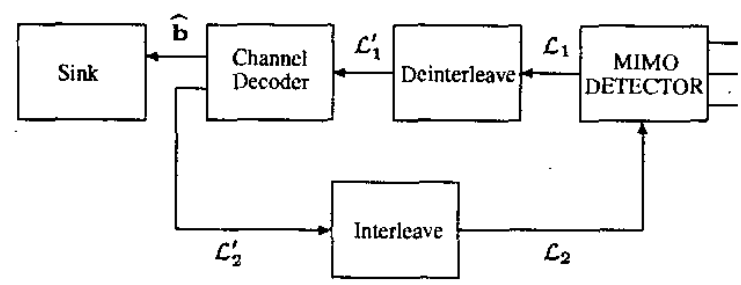

Figure 1. System model

tenna scheme with $M$ transmit and $N$ receive antennas, where the transmitted and received signal are related by

$$
x=\sqrt{\frac{\rho}{M}} H \mathbf{s}+\mathbf{v} .
$$

Furthermore, we shall assume that the channel $H$ is known to the receiver via, e.g., training.

As we pointed out earlier, a space-time code enforces time and space correlation onto the transmitted symbol sequence. This correlation is generally embedded over a number of channel uses. Therefore, we shall find it useful to model the transmission over a number of channel uses, say $T$, during which the channel remains constant. In other words, we assume that

$$
\mathbf{x}_{i}=\sqrt{\frac{\rho}{T}} H \mathbf{s}_{i}+\mathbf{v}_{i}, \quad i=1, \ldots, T,
$$

and hence we can write

$$
X=\sqrt{\frac{\rho}{M}} S H+V,
$$

where $X=\left[\begin{array}{llll}\mathbf{x}_{1} & \mathbf{x}_{2} & \ldots & \mathbf{x}_{T}\end{array}\right]^{\prime} \in \mathcal{C}^{T \times N}$ is the received signal, $S=\left[\begin{array}{llll}\mathbf{s}_{1} & \mathbf{s}_{2} & \ldots & \mathbf{s}_{T}\end{array}\right]^{]^{\prime} \in \mathcal{C}^{T \times M} \text { is the transmitted }}$ signal, and $V=\left[\begin{array}{llll}\mathbf{v}_{1} & \mathbf{v}_{2} & \ldots & \mathbf{v}_{T}\end{array}\right]^{\prime} \in \mathcal{C}^{T \times N}$ is the additive $\mathcal{C}(0,1)$ noise. Furthermore, the transmitted signal matrix satisfies the power constraint $E t r S S^{*}=T M$.

A linear-dispersion $(L D)$ code is such for which

$$
S=\sum_{q=1}^{Q}\left(s_{q} C_{q}+s_{q}^{*} D_{q}\right)
$$

where $C_{q} \in \mathcal{C}^{T \times M}$ and $D_{q} \in \mathcal{C}^{T \times M}$ are fixed matrices, and $s_{q}, q=1, \ldots, Q$ are complex scalars. Scalars $s_{q}$ can be chosen from either PSK or QAM constellations. For simplicity, we shall assume that they are chosen from a $D$-QAM constellation. The particular choice of scalars $s_{1}, \ldots, s_{Q}$ determines a specific codeword from the code that is determined by the set of matrices $\left\{C_{q}, D_{q}\right\}$. The rate of the LD code is then

$$
R=(Q / T) \log _{2} r .
$$

A reasonable choice of $Q$ is suggested in [20],

$$
Q=\min (M, N) \cdot T .
$$

Large values of $Q$ maximize the mutual information between $x$ and $s$, since the matrix $S$ then has more degrees of freedom. On the other hand, smaller $Q$ provides better coding effects - i.e., the system of linear equations that the receiver is solving becomes more overdetermined.

\subsection{Decoding of $\mathrm{LD}$ codes}

Matrix $S$ can be considered a symbol that is being transmitted (over $T$ channel uses). The total number of matrices $S$ that can be generated by $\left\{C_{q}, D_{q}\right\}$ and $s_{1}, \ldots, s_{Q}$ is $2^{R T}$ and thus can be very large. This symbol space can easily be generated by the LD codes. However, its enormous size generally prevents any exhaustive search decoding technique and asks for more sophisticated receiver algorithms. For instance, the system employing $M=8$ transmit antennas coded over $T=M$ channel uses with a rate $R=16 \mathrm{LD}$ code has a symbol space with $2^{R T} \approx 3.4 \cdot 10^{38}$ elements. Real-time exhaustive search over such a large set is out of question.

We shall find it convenient for the decoding purposes to represent the scalar $s_{q}$ by its real and imaginary parts,

$$
s_{q}=\alpha_{q}+j \beta_{q}, q=1, \ldots, Q .
$$

Denoting $A_{q}=C_{q}+D_{q}, B_{q}=C_{q}-D_{q}$, one can write

$$
S=\sum_{q=1}^{Q}\left(\alpha_{q} A_{q}+j \beta_{q} B_{q}\right)
$$

The linearity of the LD codes (4) in the variables $\left\{\alpha_{q}, \beta_{q}\right\}$ can be exploited to pose the detection problem as an integer least-squares problem. To this end, we write the equation (2) as

$$
X=\sqrt{\frac{\rho}{M}} S H+V=\sqrt{\frac{\rho}{M}} \sum_{q=1}^{Q}\left(\alpha_{q} A_{q}+j \beta_{q} B_{q}\right) H+V .
$$

Define

$$
\mathcal{A}_{q}=\left[\begin{array}{cc}
A_{R, q} & -A_{I, q} \\
A_{I, q} & A_{R, q}
\end{array}\right], \quad \mathcal{B}_{q}=\left[\begin{array}{cc}
-B_{I, q} & -B_{R, q} \\
B_{R, q} & -B_{I, q}
\end{array}\right]
$$


and

$$
\underline{h}_{n}=\left[\begin{array}{c}
h_{R, n} \\
h_{I, n}
\end{array}\right],
$$

where the vectors $h_{R, n}$ and $h_{I, n}, n=1, \ldots, N$, denote $k^{\text {th }}$ columns of matrices $H_{R}$ and $H_{I}$, respectively. Now, collecting all real and imaginary parts, we can write the expression for the input/output relation as

$$
\underbrace{\left[\begin{array}{c}
x_{R, 1} \\
x_{I, 1} \\
\vdots \\
x_{R, n} \\
x_{I, N}
\end{array}\right]}_{==}=\underbrace{\left[\begin{array}{c}
\alpha_{1} \\
\beta_{1} \\
\vdots \\
\alpha_{Q} \\
\beta_{Q}
\end{array}\right]}_{=s}+\underbrace{\left[\begin{array}{c}
v_{R, 1} \\
v_{I, 1} \\
\vdots \\
v_{R, N} \\
v_{I, N}
\end{array}\right]}_{=v},
$$

where the $2 N T \times 2 Q$ real valued equivalent channel matrix is given by

$\mathcal{H}=\sqrt{\frac{\rho}{M}} \mathcal{H}\left[\begin{array}{ccccc}\mathcal{A}_{1} \underline{h}_{1} & \mathcal{B}_{1} \underline{h}_{1} & \ldots & \mathcal{A}_{Q} \underline{h}_{1} & \mathcal{B}_{Q} \underline{h}_{1} \\ \vdots & \vdots & \ddots & \vdots & \vdots \\ \mathcal{A}_{1} \underline{h}_{N} & \mathcal{B}_{1} \underline{h}_{N} & \ldots & \mathcal{A}_{Q} \underline{h}_{N} & \mathcal{B}_{Q} \underline{h}_{N}\end{array}\right]$

and where $x_{R, k}, x_{I, k}, v_{R, k}$, and $v_{I, k}$ denote $k^{\text {th }}$ column of $X_{R}, X_{I}, V_{R}$, and $V_{I}$, respectively. Therefore, the relation between the transmitted sequence of the symbols and the received signal is linear and is given by

$$
x=\mathcal{H} s+v \text {. }
$$

The equivalent channel $\mathcal{H}$ is therefore comprised of the dispersion matrices $\left\{\mathcal{A}_{q}, \mathcal{B}_{q}\right\}$ and the known channel matrix $H$ and is, therefore, known to the receiver.

To keep the notation simple, we shall denote

$$
s_{1} \triangleq \alpha_{1}, s_{2} \triangleq \beta_{1}, \ldots, s_{2 Q-1} \triangleq \alpha_{Q}, s_{2 Q} \triangleq \beta_{Q} .
$$

Also, denote $m=2 Q$. Note that $s_{i} \in \mathcal{D}_{L}$ for $1 \leq i \leq m$, where $\mathcal{D}_{L}$ denotes an $L$-PAM constellation $(L=\sqrt{D})$. Then from (5), one can pose the maximum a posteriori detection problem as

$$
\min _{s}\|x-\mathcal{H} s\|^{2}+\sum \log p\left(s_{i}\right)
$$

where $p\left(s_{i}\right)$ is the a priori probability of the $i^{\text {th }}$ component of the $m$-dimensional vector $s$. Problem (6) allows for efficient implementation of FP-MAP algorithm [18].

\section{Modified FP Algorithm for MAP Detection}

The MAP detector maximizes the posterior probability $p_{s \mid x}(s \mid x)$, i.e., it solves optimization problem

$$
\max _{s \in \mathcal{D}_{L}^{m}} p_{s \mid x}(s \mid x)
$$

Using Bayes' rule,

$$
\arg \max _{s \in \mathcal{D}_{L}^{m}} p_{s \mid x}(s \mid x)=\arg \max _{s \in \mathcal{D}_{L}^{m}} p_{x \mid s}(x \mid s) p_{s}(s)
$$

Further, by assuming that the symbols $s_{1}, s_{2}, \ldots, s_{m}$ are independent, we can write

$$
p_{s}(s)=\prod_{k=1}^{m} p\left(s_{k}\right)=e^{\sum_{k=1}^{m} \log p\left(s_{k}\right)}
$$

Then, for a known channel in AWGN, (7) is equivalent to the optimization problem

$$
\min _{s \in \mathcal{D}_{L}^{m}}\left[\|x-\mathcal{H} s\|^{2}-\sum_{k=1}^{m} \log p\left(s_{k}\right)\right]
$$

For an iterative decoding scheme, we also require soft information, i.e., the probability that each bit is decoded correctly. To this end, consider the LIR

$$
L_{1}\left(c_{i} \mid x\right)=\log \frac{\left[\sum_{s: c_{i}=+1} e^{-\|x-\mathcal{H} s\|^{2}+\sum_{j} \log p\left[s_{j}\right]}\right]}{\left[\sum_{:: c_{i}=-1} e^{-\|x-\mathcal{H} s\|^{2}+\sum_{j} \log p\left[s_{j}\right]}\right]}
$$

Computing (9) over entire signal space $\mathcal{D}_{L}^{m}$ is of prohibitive complexity. Instead, we constrain ourselves to those $s \in$ $\mathcal{D}_{L}^{m}$ for which argument in (8) is small. [Note that these are the signal vectors whose contribution to the numerator and denominator in (9) is significant.]

Applying the idea of Fincke-Pohst algorithm, we search for the points $s$ that belong to the geometric body described by

$$
r^{2} \geq(s-\hat{s})^{*} R^{*} R(s-\hat{s})-\sum_{k=1}^{n} \log p\left(s_{k}\right),
$$

where $R$ is lower triangular matrix following QR factorization of $H$. The distance measure $r$ in (10) can be chosen according to the statistical properties of the noise and the a priori distribution of $s$. The expected complexity of the Fincke-Pohst algorithm was shown to be polynomial over a wide range of SNRs in [22].

Assume that the search yields the set of points $\mathcal{S}=$ $\left\{s^{(1)}, s^{(2)}, \ldots, s^{\left(l_{s}\right)}\right\}$. The vector $s \in \mathcal{S}$ that minimizes (8) is the solution to the MAP detection problem (7). The soft information for each bit $c_{i}$ can be estimated from (9), by only summing the terms in the numerator and denominator such that $s \in \mathcal{S}$.

Note that the logarithms in (9) can be efficiently computed using the standard Log-MAP implementation (see, e.g., [21]) of the form

$$
\begin{aligned}
\log \left(e^{\delta_{1}}+e^{\delta_{2}}\right) & =\max \left(\delta_{1}, \delta_{2}\right)+\ln \left(1+e^{-\left|\delta_{2}-\delta_{1}\right|}\right) \\
& =\max \left(\delta_{1}, \delta_{2}\right)+f_{c}\left(\left|\delta_{2}-\delta_{1}\right|\right),
\end{aligned}
$$


where $f_{\mathrm{c}}(\cdot)$ is a correction function found by a table lookup. Further computational savings (but at the expense of performance) can be obtained by dropping $f_{c}(\cdot)$ in (11), which yields Max-Log-MAP approximation of the form

$$
\log \left(e^{\delta_{1}}+e^{\delta_{2}}\right) \approx \max \left(\delta_{1}, \delta_{2}\right) .
$$

\section{Performance results}

In this section, we present examples that illustrate performance of the LDPC codes in a multi-antenna system with LD space-time modulation. In particular, we use an $8 / 9$ rate LDPC code of the length 1224 .

Figure 2 shows the performance of the $2 \times 2$ system employing a 4-QAM modulation scheme and a rate $R=4 \mathrm{LD}$ code. The significant coding gain due to use of the LDPC code is evident.

It is interesting to compare the performance of the LD code and the V-BLAST. This is shown in Figure 3. The system employing LD code outperforms the V-BLAST, with or without the channel code. However, the coding gain advantage of the LD code with respect to the V-BLAST is much more pronounced when no LDPC code is employed. Note that the dispersion matrices of the LD code are chosen to optimize the mutual information between $x$ and $s$, i.e., to solve for the optimization problem $C_{L D}(\rho, T, M, N)=$

$$
\max _{A_{q}, B_{q}, Q=1, \ldots, Q} \frac{1}{2 T} E \log \operatorname{det}\left(I_{2 N T}+\frac{\rho}{M} \mathcal{H} \mathcal{H}^{\prime}\right)
$$

for a particular $\rho$ of interest and an appropriate power constraint. It turns out that that V-BLAST satisfies this criterion as well. That explains the strong performance of V-BLAST in the system employing the LDPC code: to attain the good performance promised by a powerful channel code, all that space-time code must do is not throw away any information, which is guaranteed by satisfying (12). [There is, of course, additional gain obtained by spreading information across space and time, as illustrated in Figure 3.] Indeed, even without a space-time coding, LDPC code would yield good performance, as illustrated in Figure 4 for a system employing $M=N=4$ transmit and receive antennas and a 4-QAM modulation scheme. However, a space-time coding is needed in systems with less receive than transmit antennas, to provide as many equations as there are unknowns and provide feasibility of decoding (when $M>N$, the Fincke-Pohst algorithm is exponential in $M-N$ ). Such a system, with $M=3$ and $N=1$, employing an LD code is shown in Figure 5.

\section{Conclusion}

We studied the multi-antenna systems employing spacetime modulation and powerful channel codes. A modifi-

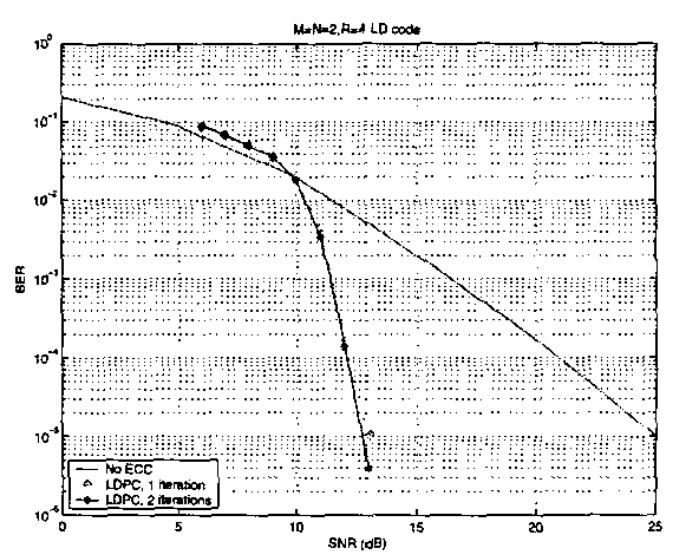

Figure 2. $M=N=2, L D R=4$, rate $8 / 9 L D P C$ code

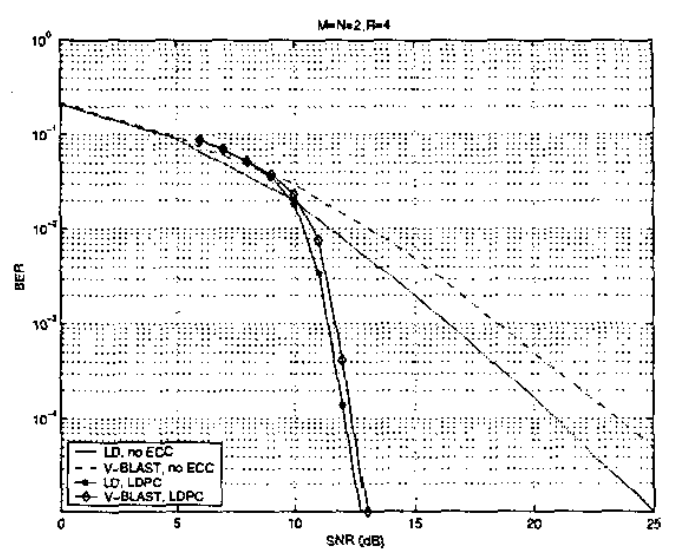

Figure 3. $M=N=2, L D$ vs. $V$-BLAST $R=4$, rate $8 / 9$ LDPC code

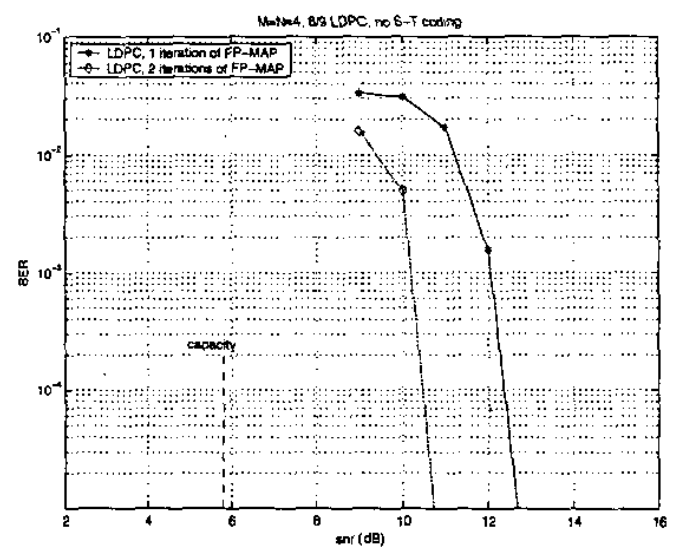

Figure 4. $M=, N=4$, no $S$ - $T$ code, rate $8 / 9 L D P C$ code 


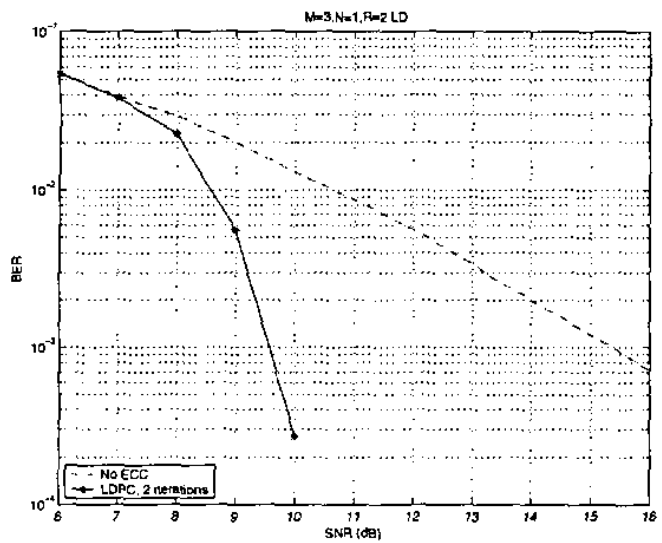

Figure 5. $M=3, N=1, R=2 L D$ code, rate $8 / 9$ LDPC code

cation of the Fincke-Pohst algorithm is used to provide an efficient estimate of the soft information required for the iterative channel decoder. Simulations illustrate the significant coding gains obtained.

\section{References}

[1] I. E. Telatar, "Capacity of multi-antenna gaussian channels," Bell Labs technical memorandum, 1995.

[2] G. J. Foschini, "Layered space-time architecture for wireless communication in a fading environment when using multielement antennas," Bell Labs. Tech. Journal, vol. 1, no. 2, pp. 41-59, 1996.

[3] A. Wittneben, "Basestation modulation diversity for digital simulcast," Proceedings IEEE VTC, pp. 848-853, 1991.

[4] N. Seshadri and J. Winters, "Two signaling schemes for improving the error performance of frequency-division-duplex (fdd) transmission systems using transmitter antenna diversity," Proceedings IEEE VTC, pp. 508-511, 1993.

[5] J. Winters, "The diversity gain of transmit fiversity in wireless systems with Rayleigh fading," Proceedings IEEE ICC, pp. 1121-1125, 1994.

[6] G. D. Golden, G. J. Foschini, R. A. Valenzuela, and P. W. Wolniansky, "Detection algorithm and initial laboratory results using V-BLAST space-time communication architecture," Electronic Letters, vol. 35, pp. 14-16, January 1999.

[7] G. J. Foschini, G. D. Golden, R. A. Valenzuela, and P. Wolniansky, "Simplified processing for high spectral efficiency wireless communication employing multi-antenna arrays," IEEE Journal on Selected Areas in Communications, vol. 17, pp. 1841-1852, November 1999.

[8] V. Tarokh V, N. Seshadri, and A. R. Calderbank, "Space-time Codes for High Data Rate Wireless Communication: Performance Criterion and Code Construction," IEEE Transactions on Information Theory, Vol. 44, No. 2, pp. 744-765, Mar. 1998.

[9] S. M. Alamouti, "A simple transmitter diversity scheme for wireless communications," IEEE Journal on Selected Areas in Communications, pp. 1451-1458, October 1998.

[10] V. Tarokh, H. Jafarkhani, and A. R. Calderbank, "Space-time block codes from orthogonal designs," IEEE Transactions on Information Theory, vol. 45, pp. 1456-1467, July 1999.

[11] B. M. Hochwald and T. L. Marzetta, "Unitary space-time modulation for multiple-antenna communication in Rayleigh flat-fading," IEEE Transactions on Information Theory, vol. 46, pp. 543-564, March 2000.

[12] B. Hochwald and W. Sweldens, "Differential unitary spacetime modulation," IEEE Transactions on Communications, vol. 48, pp. 2041-2052, December 2000 .

[13] B. L. Hughes, "Differential space-time modulation," IEEE Transactions on Information Theory, pp. 2567-2578, November 2000.

[14] A. Shokrollahi, B. Hassibi, B. Hochwald, and W. Sweldens, "Representation theory for high-rate multiple-antenna code design," IEEE Transactions on Information Theory, pp. 2567-2578, November 2000.

[15] V. Tarokh and H. Jafarkhani, "A differential detection scheme for transmit diversity," IEEE Joumal on Selected Areas in Communications, pp. 1169-1174, July 2000.

[16] B. Hassibi and B. Hochwald, "Cayley differential unitary space-time codes," IEEE Transactions on Information theory.

[17] A. Stefanov and T. M. Duman, "Turbo-coded modulation for systems with transmit and receive antenna diversity over block fading channels: system model, decoding approaches, and practical considerations," IEEE Joumal on Sel. Areas in Commun., vol. 19, no. 5, May 2001.

[18] H. Vikalo and B. Hassibi, "Iterative decoding over multiple antenna channels via a modified sphere decoder," Proceedings of $39^{\text {th }}$ Allerton conference on Communication, Control, and Computing, Allerton, $\mathrm{LL}, 2001$.

[19] B. M. Hochwald and S. ten Brink, "Achieving near-capacity on a multiple-antenna channel," Proceedings of $39^{\text {th }}$ Allerton conference on Communication, Control, and Computing, Allerton, IL, 2001.

[20] B. Hassibi and B. Hochwald, "High-rate codes that are linear in space and time," submitted to IEEE Trans. Info. Theory, 2000. Download available at http://mars bel1labs.com

[21] P. Robertson, P. Hoeher and E. Villebrun, "Optimal and suboptimal maximum a posteriori algorithms suitable for turbo decoding", European Trans. Telecommun., vol. 8, pp. 119125, Mar-Apr. 1997.

[22] B. Hassibi and H. Vikalo, "Expected complexity of the sphere decoder algorithm," submitted to the IEEE Transactions on Signal Processing, 2002. 